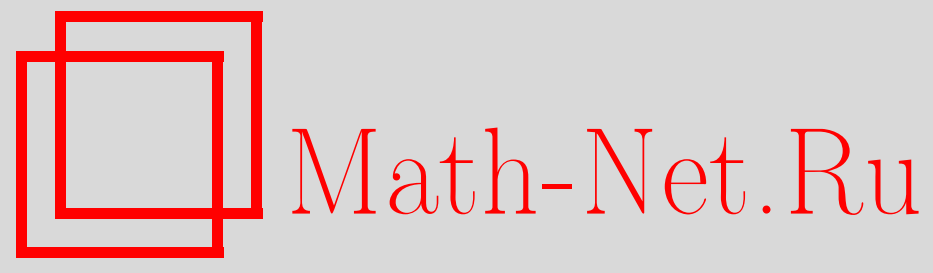

А. В. Тарасенко, И. П. Егорова, О нелокальной задаче с дробной производной Римана-Лиувилля для уравнения смешанного типа, Вестн. Сам. гос. техн. ун-та. Сер. Физ.-мат. науки, 2017, номер 1, 112-121

DOI: https://doi.org/10.14498/vsgtu1499

Использование Общероссийского математического портала MathNet.Ru подразумевает, что вы прочитали и согласны с пользовательским соглашением

http://www.mathnet.ru/rus/agreement

Параметры загрузки:

IP : 54.209 .52 .79

26 апреля 2023 г., 18:07:16

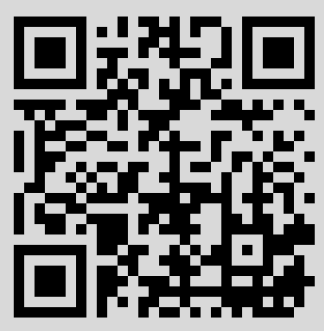


Вестн. Сам. гос. техн. ун-та. Сер. Физ.-мат. науки. 2017. Т. 21, № 1. С. $112-121$ ISSN: 2310-7081 (online), 1991-8615 (print)

УДК 517.956.6

\section{О нелокальной задаче с дробной производной Римана-Лиувилля для уравнения смешанного типа}

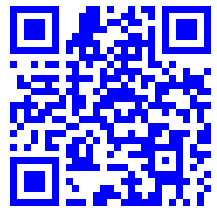

\section{А. В. Тарасенко, И. П. Егорова}

Самарский государственный технический университет,

Россия, 443100, Самара, ул. Молодогвардейская, 244.

\section{Аннотация}

Для уравнения с частной дробной производной Римана-Лиувилля исследована однозначная разрешимость задачи с обобщенным оператором дробного интегро-дифференцирования в краевом условии. Теорема единственности решения поставленной задачи доказана на основании принципа экстремума для нелокального параболического уравнения и принципа экстремума для операторов дробного дифференцирования в смысле Римана-Лиувилля. Доказательство существования решения эквивалентно сводится к вопросу разрешимости дифференциального уравнения дробного порядка. Решение рассматриваемой задачи получено в явном виде.

Ключевые слова: краевая задача, обобщенный оператор дробного интегро-дифференцирования, гипергеометрическая функция Гаусса, дифференциальное уравнение дробного порядка.

Получение: 30 июня 2016 г. / Исправление: 8 октября 2016 г.

Принятие: 9 декабря 2016 г. / Публикация онлайн:

1. Постановка задачи. Рассмотрим уравнение

$$
\begin{cases}u_{x x}-D_{0+, y}^{\alpha} u=0, & y>0 \\ (-y)^{m} u_{x x}-u_{y y}+a(-y)^{\frac{m}{2}-1} u_{x}=0, & y<0\end{cases}
$$

где $D_{0+, y}^{\alpha}-$ частная дробная производная Римана-Лиувилля от функции $u(x, y)$ порядка $\alpha(0<\alpha<1)[1$, с. 42,44$]$ :

$$
\left(D_{0+, y}^{\alpha} u\right)(x, y)=\left(\frac{\partial}{\partial y}\right) \frac{1}{\Gamma(1-\alpha)} \int_{0}^{y} \frac{u(x, t) d t}{(y-t)^{\alpha}}, \quad 0<\alpha<1, \quad y>0,
$$

\section{Статья}

(2)(7) Контент публикуется на условиях лицензии Creative Commons Attribution 4.0 International (https://creativecommons.org/licenses/by/4.0/deed.ru)

\section{Образец для цитирования}

Т а р а с ен ко А. В., Е г о р в в И. П. О нелокальной задаче с дробной производной РиманаЛиувилля для уравнения смешанного типа // Вестн. Сам. гос. техн. ун-та. Сер. Физ.мат. науки, 2017. Т. 21, № 1. C. 112-121. doi: 10.14498/vsgtu1499.

\section{Сведения об авторах}

Анна Валерьевна Тарасенко (D) http://orcid.org/0000-0002-0487-8262

кандидат физико-математических наук, доцент; доцент; каф. высшей математики АСИ СамГТУ; e-mail: tarasenko.a.v@mail.ru

Ирина Петровна Егорова (D) http://orcid.org/0000-0001-6023-1466

кандидат физико-математических наук, доцент; доцент; каф. высшей математики АСИ СамГТУ; e-mail: ira.egorova81@yandex.ru 
$a$ - вещественная постоянная, $m>2$, в конечной области $\Omega$, ограниченной отрезками $A A_{0}, B B_{0}, A_{0} B_{0}$ прямых $x=0, x=1, y=1$ соответственно, лежащих в полуплоскости $y>0$, и характеристиками

$$
A C: x-\frac{2}{m+2}(-y)^{\frac{m+2}{2}}=0, \quad B C: x+\frac{2}{m+2}(-y)^{\frac{m+2}{2}}=1
$$

уравнения (1) в полуплоскости $y<0$.

Пусть $\Omega_{1}=\Omega \cap(y>0), \Omega_{2}=\Omega \cap(y<0), I \equiv A B$ - единичный интервал $0<x<1$ прямой $y=0$.

ЗАДАчА.Найти решение $u(x, y)$ уравнения (1) в области $\Omega$ при $y \neq 0$, удовлетворяющее краевым условиям

$$
\begin{gathered}
u(0, y)=\varphi_{1}(y), \quad u(1, y)=\varphi_{2}(y), \quad 0 \leqslant y \leqslant 1, \\
A_{1}\left(I_{0+}^{-\beta^{*}, 0, \beta^{*}+\beta-1} u\left[\Theta_{0}(t)\right]\right)(x)+A_{2} u(x, 0)=A_{3} g(x),
\end{gathered}
$$

а такюе условиям сопряюения

$$
\begin{gathered}
\lim _{y \rightarrow 0+} y^{1-\alpha} u(x, y)=\lim _{y \rightarrow 0-} u(x, y), \quad \forall x \in \bar{I}, \\
\lim _{y \rightarrow 0+} y^{1-\alpha}\left(y^{1-\alpha} u(x, y)\right)_{y}=\lim _{y \rightarrow 0-} u_{y}(x, y), \quad \forall x \in I,
\end{gathered}
$$

где $A_{i}(i=1,2,3)$ - действительные константы такие, что

$$
-\frac{\Gamma(\beta) A_{2}}{\Gamma\left(\beta^{*}+\beta\right)}<A_{1}<0 \quad \text { либо } \quad 0<A_{1}<-\frac{\Gamma(\beta) A_{2}}{\Gamma\left(\beta^{*}+\beta\right)},
$$

$\varphi_{i}(y)(i=1,2), g(x)$ - заданные функиии такие, что

$$
\begin{gathered}
g(x) \in C^{1}(\bar{I}) \cap C^{3}(I), \\
\varphi_{1}(0)=\varphi_{2}(0)=0 \\
y^{1-\alpha} \varphi_{1}(y), \quad y^{1-\alpha} \varphi_{2}(y) \in C([0,1]),
\end{gathered}
$$

$\Theta_{0}(x)$ - точка пересечения характеристик уравнения (1), выходящих из точки $(x, 0) \in I$, с характеристикой $A C$;

$$
\beta^{*}=\frac{m-2 a}{2(m+2)}, \quad \beta=\frac{m+2 a}{2(m+2)}, \quad|a|<\frac{m}{2}, \quad 0<\beta^{*}<\frac{1}{2}, \quad 0<\beta<\frac{1}{2} .
$$

Будем искать решение $u(x, y)$ поставленной задачи в классе дважды дифференцируемых функций в области $\Omega$ таких, что

$$
\begin{aligned}
& y^{1-\alpha} u(x, y) \in C\left(\bar{\Omega}_{1}\right), \quad u(x, y) \in C\left(\bar{\Omega}_{2}\right), \\
& y^{1-\alpha}\left(y^{1-\alpha} u\right)_{y} \in C\left(\Omega_{1} \cup\{(x, y): 0<x<1, y=0\}\right), \\
& u_{x x} \in C\left(\Omega_{1} \cup \Omega_{2}\right), \quad u_{y y} \in C\left(\bar{\Omega}_{2}\right) .
\end{aligned}
$$

$\left(I_{0+}^{\alpha, \beta, \eta} f\right)(x)$ - оператор обобщенного дробного интегро-дифференцирования с гипергеометрической функцией Гаусса $F(a, b ; c ; z)$, введенный М. Сайго [2] 
(см. также [1, с. 326-327]) и имеющий при действительных $\alpha, \beta, \eta$ и $x>0$ вид

$$
\left(I_{0+}^{\alpha, \beta, \eta} f\right)(x)=\left\{\begin{array}{l}
\frac{x^{-\alpha-\beta}}{\Gamma(\alpha)} \int_{0}^{x}(x-t)^{\alpha-1} F\left(\alpha+\beta,-\eta ; \alpha ; 1-\frac{t}{x}\right) f(t) d t, \alpha>0, \\
\left(\frac{d}{d x}\right)^{n}\left(I_{0+}^{\alpha+n, \beta-n, \eta-n} f\right)(x), \alpha \leqslant 0, n=[-\alpha]+1,
\end{array}\right.
$$

Заметим, что если $\alpha>0$, то справедливы формулы

$$
\left(I_{0+}^{\alpha,-\alpha, \eta} f\right)(x)=\left(I_{0+}^{\alpha} f\right)(x), \quad\left(I_{0+}^{-\alpha, \alpha, \eta} f\right)(x)=\left(D_{0+}^{\alpha} f\right)(x),
$$

в частности

$$
\left(I_{0+}^{0,0, \eta} f\right)(x)=f(x), \quad\left(I_{1-}^{0,0, \eta} f\right)(x)=f(x),
$$

где $\left(I_{0+}^{\alpha} f\right)(x)$ и $\left(D_{0+}^{\alpha} f\right)(x)$ - операторы дробного интегрирования и дифференцирования Римана-Лиувилля порядка $\alpha>0[1$, c. 42,44$]$ :

$$
\begin{aligned}
& \left(I_{0+}^{\alpha} f\right)(x)=\frac{1}{\Gamma(\alpha)} \int_{0}^{x}(x-t)^{\alpha-1} f(t) d t, \quad \alpha>0, x>0 \\
& \left(D_{0+}^{\alpha} f\right)(x)=\left(\frac{d}{d x}\right)^{n} \frac{1}{\Gamma(n-\alpha)} \int_{0}^{x}(x-t)^{n-\alpha-1} f(t) d t, \quad \alpha>0, n=[\alpha]+1 .
\end{aligned}
$$

Истоком настоящей задачи послужили публикации $[3,4]$.

2. Единственность решения задачи. Пусть существует решение исследуемой задачи. Введем обозначения

$$
\begin{gathered}
\lim _{y \rightarrow 0+} y^{1-\alpha} u(x, y)=\tau_{1}(x), \quad \lim _{y \rightarrow 0-} u(x, y)=\tau_{2}(x), \\
\lim _{y \rightarrow 0+} y^{1-\alpha}\left(y^{1-\alpha} u(x, y)\right)_{y}=\nu_{1}(x), \quad \lim _{y \rightarrow 0-} u_{y}(x, y)=\nu_{2}(x) .
\end{gathered}
$$

Известно (см., например, $[5,6]$ ), что решение уравнения $(1)$ в области $\Omega_{1}$, удовлетворяющее условию (2) и условию

$$
\lim _{y \rightarrow 0+} y^{1-\alpha} u(x, y)=\tau_{1}(x), \quad x \in \bar{I},
$$

дается формулой

$$
\begin{aligned}
u(x, y)=\int_{0}^{y} \varphi_{1}(\eta) G_{\xi}(x, y, 0, \eta) d \eta- & \\
& \quad-\int_{0}^{y} \varphi_{2}(\eta) G_{\xi}(x, y, 1, \eta) d \eta+\Gamma(\alpha) \int_{0}^{1} \tau_{1}(\xi) G(x, y, \xi, 0) d \xi,
\end{aligned}
$$

где

$$
G(x, y, \xi, \eta)=\frac{(y-\eta)^{\beta-1}}{2} \sum_{n=-\infty}^{\infty}\left\{e_{1, \beta}^{1, \beta}\left(-\frac{|x-\xi+2 n|}{(y-\eta)^{\beta}}\right)-\right.
$$




$$
\begin{array}{r}
\left.-e_{1, \beta}^{1, \beta}\left(-\frac{|x+\xi+2 n|}{(y-\eta)^{\beta}}\right)\right\}, \quad \beta=\frac{\alpha}{2}, \\
e_{b, c}^{p, q}(z)=\sum_{k=0}^{\infty} \frac{z^{k}}{\Gamma(b k+p) \Gamma(q-c k)}, \quad b>c, \quad b>0, \quad z \in \mathbb{C} .
\end{array}
$$

Также известно (см., например, [5]), что функциональное соотношение между $\tau_{1}(x)$ и $\nu_{1}(x)$, принесенное из параболической части $\Omega_{1}$ на линию $y=0$, имеет вид

$$
\nu_{1}(x)=\frac{1}{\Gamma(1+\alpha)} \tau_{1}^{\prime \prime}(x) .
$$

Найдем соотношение между $\tau_{2}(x)$ и $\nu_{2}(x)$, принесенное на линию $y=0$ из гиперболической части $\Omega_{2}$ области $\Omega$. Для этого воспользуемся решением задачи Коши, которое в области $\Omega_{2}$ имеет вид [7]

$$
\begin{aligned}
u(x, y)= & \frac{\Gamma\left(\beta^{*}+\beta\right)}{\Gamma\left(\beta^{*}\right) \Gamma(\beta)} \times \\
& \times \int_{0}^{1} \tau_{2}\left(x+\frac{2}{m+2}(-y)^{\frac{m+2}{2}}(2 t-1)\right) t^{\beta-1}(1-t)^{\beta^{*}-1} d t+ \\
& \quad+\frac{\Gamma\left(2-\beta^{*}-\beta\right)}{\Gamma\left(1-\beta^{*}\right) \Gamma(1-\beta)}(-y) \times \\
& \quad \times \int_{0}^{1} \nu_{2}\left(x+\frac{2}{m+2}(-y)^{\frac{m+2}{2}}(2 t-1)\right) t^{-\beta^{*}}(1-t)^{-\beta} d t .
\end{aligned}
$$

Используя формулу (14) и соотношения (8), получим

$$
\begin{aligned}
u\left[\Theta_{0}(x)\right]=\frac{\Gamma\left(\beta^{*}+\beta\right)}{\Gamma(\beta)} & \left(I_{0+}^{\beta^{*}, 0, \beta-1} \tau_{2}(t)\right)(x)+ \\
+ & \frac{\Gamma\left(2-\beta^{*}-\beta\right)}{\Gamma\left(1-\beta^{*}\right)}\left(\frac{m+2}{4}\right)^{\frac{2}{m+2}}\left(I_{0+}^{1-\beta, \beta^{*}+\beta-1, \beta-1} \nu_{2}(t)\right)(x) .
\end{aligned}
$$

Подставляя $u\left[\Theta_{0}(x)\right]$ в краевое условие $(3)$, учитывая $(9)$ и $(10)$, получим

$$
\begin{aligned}
& \left(A_{1} \frac{\Gamma\left(\beta^{*}+\beta\right)}{\Gamma(\beta)}+A_{2}\right) \tau_{2}(x)+ \\
& \quad+A_{1} \frac{\Gamma\left(2-\beta^{*}-\beta\right)}{\Gamma\left(1-\beta^{*}\right)}\left(\frac{m+2}{4}\right)^{\frac{2}{m+2}}\left(I_{0+}^{1-\beta-\beta^{*}} \nu_{2}(t)\right)(x)=A_{3} g(x) .
\end{aligned}
$$

Применив к обеим частям полученного равенства оператор $D_{0+}^{1-\beta-\beta^{*}}$, с учеTOM

$$
\left(D_{0+}^{\alpha}\left(I_{0+}^{\alpha} f\right)(t)\right)(x)=f(x), \quad \alpha>0
$$

будем иметь

$$
\begin{aligned}
\left(A_{1} \frac{\Gamma\left(\beta^{*}+\beta\right)}{\Gamma(\beta)}\right. & \left.+A_{2}\right)\left(D_{0+}^{1-\beta-\beta^{*}} \tau_{2}(t)\right)(x)+ \\
& +A_{1} \frac{\Gamma\left(2-\beta^{*}-\beta\right)}{\Gamma\left(1-\beta^{*}\right)}\left(\frac{m+2}{4}\right)^{\frac{2}{m+2}} \nu_{2}(x)=A_{3}\left(D_{0+}^{1-\beta-\beta^{*}} g(t)\right)(x) .
\end{aligned}
$$


Рассмотрим соответствующую однородную задачу: $g(x)=0$.

Выразим из последнего выражения $\nu_{2}(x)$, в результате получим

$$
\nu_{2}(x)=\lambda_{1}\left(D_{0+}^{1-\beta-\beta^{*}} \tau_{2}(t)\right)(x)
$$

где

$$
\lambda_{1}=-\frac{A_{1} \frac{\Gamma\left(\beta^{*}+\beta\right)}{\Gamma(\beta)}+A_{2}}{A_{1} \frac{\Gamma\left(2-\beta^{*}-\beta\right)}{\Gamma\left(1-\beta^{*}\right)}\left(\frac{m+2}{4}\right)^{\frac{2}{m+2}}} .
$$

Докажем две леммы.

ЛЕмма 1. Если бункиия $\tau_{1}(x)$ достигает положителъного максимума (отрицательного минимума) на отрезке $[0,1]$ в точке $x=x_{0}\left(0<x_{0}<1\right)$, mo $\nu_{1}\left(x_{0}\right) \leqslant 0\left(\nu_{1}\left(x_{0}\right) \geqslant 0\right)$.

Лемма 2. Если функиия $\tau_{2}(x)$ достигает положителъного максимума (отрицательного минимума) на отрезке $[0,1]$ в точке $x=x_{0}\left(0<x_{0}<1\right)$ $u$ выполняются условия $g(x)=0 u(6)$, то $\nu_{2}\left(x_{0}\right)>0\left(\nu_{2}\left(x_{0}\right)<0\right)$.

Справедливость леммы 1 непосредственно следует из соотношения (13).

Справедливость леммы 2 вытекает из соотношения (16), условий (6) и $g(x)=0$ и свойства строгой положительности (строгой отрицательности) производной дробного порядка $\left(D_{0+}^{\gamma} \tau_{2}(t)\right)(x)$ в точке положительного максимума (отрицательного минимума) [8, с. 105].

Справедлива следующая теорема.

ТЕорема 1. Пусть выполняются неравенства (6) и условия сопряжения (4), (5). Тогда, если существует решение исследуемой задачи, то оно единственно.

Доказательство. Используем ранее введенные обозначения точек $A_{0}(0,1)$ и $B_{0}(1,1)$. Пусть $u(x, y)$ - решение однородной задачи, но $u(x, y) \neq 0$ в области $\widetilde{\Omega}=\Omega_{1} \cup \overline{A B} \cup \overline{A A_{0}} \cup \overline{A_{0} B_{0}} \cup \overline{B B_{0}}$. Пусть точка $Q$ - точка положительного максимума и точка $Q \in \widetilde{\Omega}$.

В силу однородных условий точка $Q$ не может принадлежать $\overline{A A_{0}} \cup \overline{B B_{0}}$. На основании принципа экстремума для нелокального параболического уравнения [9, с. 47] точка $Q$ не может принадлежать $\Omega_{1} \cup \overline{A_{0} B_{0}}$ и поэтому точка $Q \in I=A B$, точка $Q\left(x_{0}, 0\right)$.

По лемме 1

$$
\nu_{1}\left(x_{0}\right) \leqslant 0, \quad x_{0} \in I .
$$

Так как $\tau_{1}(x)=\tau_{2}(x)=\tau(x)$,

$$
\max _{\bar{I}} \tau(x)=\tau\left(x_{0}\right)
$$

В силу условий (2), (11) по лемме 2 имеем

$$
\nu_{2}\left(x_{0}\right)>0
$$

что противоречит условию (5). 
Следовательно, $u(x, y) \equiv 0$ в $\overline{\Omega_{1}}$ и, в частности, $\tau_{1}\left(x_{0}\right)=0$ на $I$. Но тогда из равенства $\tau_{1}(x)=\tau_{2}(x)$ следует, что $\nu_{2}(x)=0$.

Отсюда по формуле $(14)$ получаем, что $u(x, y) \equiv 0$ и в области $\overline{\Omega_{2}}$.

3. Существование решения задачи. Продифференцируем обе части (15) по $x$ и учтем

$$
\left(D_{0+}^{\alpha} \varphi\right)(x)=\left(I_{0+}^{-\alpha, \alpha, \eta} \varphi\right)(x)=\left(\frac{d}{d x}\right)^{n}\left(I_{0+}^{n-\alpha} \varphi\right)(x)
$$

в результате получим

$$
\begin{aligned}
\left(A_{1} \frac{\Gamma\left(\beta^{*}+\beta\right)}{\Gamma(\beta)}\right. & \left.+A_{2}\right) \tau_{2}^{\prime \prime}(x)+ \\
& +A_{1} \frac{\Gamma\left(2-\beta^{*}-\beta\right)}{\Gamma\left(1-\beta^{*}\right)}\left(\frac{m+2}{4}\right)^{\frac{2}{m+2}}\left(D_{0+}^{1+\beta+\beta^{*}} \nu_{2}(t)\right)(x)=A_{3} g^{\prime \prime}(x)
\end{aligned}
$$

Полагая $\tau_{1}(x)=\tau_{2}(x)=\tau(x), \nu_{1}(x)=\nu_{2}(x)=\nu(x)$ и учитывая $(13)$, получим дифференциальное уравнение дробного порядка $1+\beta+\beta^{*}$ :

$$
\left(D_{0+}^{1+\beta+\beta^{*}} \nu(t)\right)(x)=\lambda_{2} \nu(x)+\lambda_{3} g^{\prime \prime}(x),
$$

где

$$
\lambda_{2}=-\frac{\left(A_{1} \frac{\Gamma\left(\beta^{*}+\beta\right)}{\Gamma(\beta)}+A_{2}\right) \Gamma(1+\alpha)}{A_{1} \frac{\Gamma\left(2-\beta^{*}-\beta\right)}{\Gamma\left(1-\beta^{*}\right)}\left(\frac{m+2}{4}\right)^{\frac{2}{m+2}}}, \quad \lambda_{3}=-\frac{A_{3}}{A_{1} \frac{\Gamma\left(2-\beta^{*}-\beta\right)}{\Gamma\left(1-\beta^{*}\right)}\left(\frac{m+2}{4}\right)^{\frac{2}{m+2}}} .
$$

Известно [1], что общее решение дифференциального уравнения дробного порядка $\alpha>0$

$$
\left(D_{0+}^{\alpha} y(t)\right)(x)-\lambda y(x)=h(x), \quad \alpha>0, \quad n=-[-\alpha]
$$

дается формулой

$$
y(x)=\sum_{k=1}^{n} c_{k} x^{\alpha-k} E_{\alpha, 1+\alpha-k}\left(\lambda x^{\alpha}\right)+\int_{0}^{x}(x-t)^{\alpha-1} E_{\alpha, \alpha}\left(\lambda(x-t)^{\alpha}\right) h(t) d t .
$$

Здесь $c_{1}, c_{2}, \ldots, c_{n}$ - произвольные постоянные, а функции $E_{\alpha, 1+\alpha-k}\left(\lambda x^{\alpha}\right)$ и $E_{\alpha, \alpha}\left(\lambda(x-t)^{\alpha}\right)$ - специальные случаи функции Миттаг-Леффлера $E_{\alpha, \beta}(z)$, определяемой равенством

$$
E_{\alpha, \beta}(z)=\sum_{m=0}^{\infty} \frac{z^{m}}{\Gamma(\alpha m+\beta)}, \quad \alpha>0, \quad \beta>0 ; \quad E_{\alpha}(z) \equiv E_{\alpha, 1}(z)
$$

и являющейся целой функцией от $z$ [1]. 
Уравнение (17) - уравнение вида (18) с $y(x)=\nu(x), \alpha=1+\beta+\beta^{*}, \lambda=\lambda_{2}$ и $h(x)=\lambda_{3} g^{\prime \prime}(x)$. Так как $1<1+\beta+\beta^{*}<2$, общее решение вида (19) для уравнения (17) дается формулой

$$
\begin{gathered}
\nu(x)=c_{1} x^{\beta+\beta^{*}} E_{1+\beta+\beta^{*}, 1+\beta+\beta^{*}}\left(\lambda_{1} x^{1+\beta+\beta^{*}}\right)+ \\
\quad+c_{2} x^{\beta+\beta^{*}-1} E_{1+\beta+\beta^{*}, \beta+\beta^{*}}\left(\lambda_{1} x^{1+\beta+\beta^{*}}\right)+ \\
+\lambda_{2} \int_{0}^{x}(x-t)^{\beta+\beta^{*}} E_{1+\beta+\beta^{*}, 1+\beta+\beta^{*}}\left(\lambda_{1}(x-t)^{1+\beta+\beta^{*}}\right) g^{\prime \prime}(t) d t,
\end{gathered}
$$

где $c_{1}, c_{2}$ - произвольные постоянные.

Подставляя $(20)$ в $(15)\left(\right.$ с $\left.\tau_{2}=\tau, \nu_{2}=\nu\right)$, получаем выражение для $\tau(x)$ :

$$
\begin{gathered}
\tau(x)=c_{1}^{*}\left(I_{0+}^{1-\beta-\beta^{*}} t^{\beta+\beta^{*}} E_{1+\beta+\beta^{*}, 1+\beta+\beta^{*}}\left(\lambda_{1} t^{1+\beta+\beta^{*}}\right)\right)(x)+ \\
+c_{2}^{*}\left(I_{0+}^{1-\beta-\beta^{*}} t^{\beta+\beta^{*}-1} E_{1+\beta+\beta^{*}, \beta+\beta^{*}}\left(\lambda_{1} t^{1+\beta+\beta^{*}}\right)\right)(x)+ \\
+\lambda_{4}\left(I_{0+}^{1-\beta-\beta^{*}} \int_{0}^{t}(t-s)^{\beta+\beta^{*}} E_{1+\beta+\beta^{*}, 1+\beta+\beta^{*}}\left(\lambda_{1}(t-s)^{1+\beta+\beta^{*}}\right) \lambda_{2} g^{\prime \prime}(s) d s\right)(x)+ \\
+\frac{A_{3} \Gamma(\beta)}{A_{1} \Gamma\left(\beta+\beta^{*}\right)+A_{2} \Gamma(\beta)} g(x), \quad(21)
\end{gathered}
$$

где

$$
\begin{gathered}
\lambda_{4}=-\frac{\Gamma\left(2-\beta-\beta^{*}\right)}{\Gamma\left(1-\beta^{*}\right)} \frac{A_{1} \Gamma(\beta)}{A_{1} \Gamma\left(\beta+\beta^{*}\right)+A_{2} \Gamma(\beta)}\left(\frac{m+2}{4}\right)^{\frac{2}{m+2}}, \\
c_{1}^{*}=\lambda_{4} c_{1}, \quad c_{2}^{*}=\lambda_{4} c_{2} .
\end{gathered}
$$

Для приведения выражения (21) к более простому виду воспользуемся двумя леммами, доказанными в работе [3].

Лемма 3. Если $0<\beta<1 / 2, \lambda \in \mathbb{C}$, mo

$$
\left(I_{0+}^{1-2 \beta} t^{2 \beta} E_{2 \beta+1,2 \beta+1}\left(\lambda t^{2 \beta+1}\right)\right)(x)=x E_{2 \beta+1,2}\left(\lambda x^{2 \beta+1}\right)
$$

$u$

$$
\left(I_{0+}^{1-2 \beta} t^{2 \beta-1} E_{2 \beta+1,2 \beta}\left(\lambda t^{2 \beta+1}\right)\right)(x)=E_{2 \beta+1,1}\left(\lambda x^{2 \beta+1}\right) \equiv E_{2 \beta+1}\left(\lambda x^{2 \beta+1}\right) .
$$

Лемма 4. Если $0<\beta<1 / 2, \lambda \in \mathbb{C}$, mo

$$
\begin{aligned}
\left(I_{0+}^{1-2 \beta} \int_{0}^{t}(t-s)^{2 \beta} E_{2 \beta+1,2 \beta+1}\left(\lambda(t-s)^{2 \beta+1}\right) h(s) d s\right)(x)= & \\
& =\int_{0}^{x}(x-s) E_{2 \beta+1,2}\left(\lambda(x-s)^{2 \beta+1}\right) h(s) d s .
\end{aligned}
$$

Полагая $2 \beta=\beta+\beta^{*}, \lambda=\lambda_{1}, h(s)=\lambda_{2} g^{\prime \prime}(s)$, из равенства (21) находим формулу для $\tau(x)$ : 


$$
\begin{gathered}
\tau(x)=c_{1}^{*} x E_{1+\beta+\beta^{*}, 2}\left(\lambda_{1} x^{1+\beta+\beta^{*}}\right)+c_{2}^{*} E_{1+\beta+\beta^{*}}\left(\lambda_{1} x^{1+\beta+\beta^{*}}\right)+ \\
+\lambda_{4} \int_{0}^{x}(x-t) E_{1+\beta+\beta^{*}, 2}\left(\lambda_{1}(x-t)^{1+\beta+\beta^{*}}\right) \lambda_{2} g^{\prime \prime}(s) d s+ \\
+\frac{A_{3} \Gamma(\beta)}{A_{1} \Gamma\left(\beta+\beta^{*}\right)+A_{2} \Gamma(\beta)} g(x) .
\end{gathered}
$$

Для нахождения постоянных $c_{1}^{*}, c_{2}^{*}$ мы можем использовать соотношение $\tau(0)=\tau(1)=0$, вытекающее из условия (7). Подставляя $x=0$ в $(22)$ и учитывая вытекающее из (18) равенство $E_{1+\beta+\beta^{*}}(0)=1$, получим

$$
c_{2}^{*}=\lambda_{5} g(0), \quad \lambda_{5}=-\frac{A_{3} \Gamma(\beta)}{A_{1} \Gamma\left(\beta^{*}+\beta\right)+A_{2} \Gamma(\beta)} .
$$

Подставляя $x=1$ в формулу (22) и учитывая выражение (23), находим

$$
\begin{aligned}
c_{1}^{*}=\frac{1}{E_{1+\beta+\beta^{*}, 2}\left(\lambda_{1}\right)}( & \lambda_{5} g(1)-\lambda_{5} g(0) E_{1+\beta+\beta^{*}}\left(\lambda_{1}\right)- \\
& \left.-\lambda_{2} \lambda_{4} \int_{0}^{1}(1-s) E_{1+\beta+\beta^{*}, 2}\left(\lambda_{1}(1-s)^{1+\beta+\beta^{*}}\right) g(s) d s\right) .
\end{aligned}
$$

Таким образом, подставляя (22) в формулу (12) (с $\left.\tau_{1}=\tau\right)$, получим явное решение $u(x, y)$ исследуемой задачи. Это завершает доказательство существования решения исходной задачи.

Конкурирующие интересы. Мы не имеем конкурирующих интересов.

Авторский вклад и ответственность. Все авторы принимали участие в разработке концепции статьи и в написании рукописи. Авторы несут полную ответственность за предоставление окончательной рукописи в печать. Окончательная версия рукописи была одобрена всеми авторами.

Финансирование. Исследование не имело спонсорской поддержки.

\section{Библиографический список}

1. Самко С. Г., Килбас А. А., Маричев О. И. Интегралы и производные дробного порядка и некоторые их приложения. Минск: Наука и техника, 1987. 688 с.

2. Saigo M. A remark on integral operators involving the Gauss hypergeometric function // Math. Rep. Coll. Gen. Educ., Kyushu Univ., 1978. vol.11, no. 2. pp. 135-143.

3. Килбас А. А., Репин О. А. Аналог задачи Бицадзе-Самарского для уравнения смешанного типа с дробной производной // Дифферени. уравнения, 2003. Т. 39, № 5. С. 638-644.

4. Геккиева С. Х. Аналог задачи Трикоми для уравнения смешанного типа с дробной производной // Известия КБНЦРАН, 2001. № 2(7). С. 78-80.

5. Килбас А. А., Репин О. А. Аналог задачи Трикоми для дифференциального уравнения с частными производными, содержащего уравнение диффузии дробного порядка // Докл. АМАН, 2010. Т. 12, №1. С. 31-39.

6. Псху А. В. Уравнения в частных производных дробного порядка. М.: Наука, 2005. 199 с.

7. Смирнов М. М. Вырождающиеся эллиптические и гиперболические уравнения. М.: Наука, 1966. 292 с.

8. Нахушев А. М. Дробное исчисление его применение. М.: Физматлит, 2009. 272 с.

9. Нахушева В. А. Дифференциальные уравнения математических моделей нелокальных прочессов. М.: Наука, 2006. 173 с. 


\title{
On nonlocal problem with fractional Riemann-Liouville derivatives for a mixed-type equation
}

\author{
A. V. Tarasenko, I. P. Egorova \\ Samara State Technical University, \\ 244, Molodogvardeyskaya st., Samara, 443100, Russian Federation.
}

\begin{abstract}
The unique solvability is investigated for the problem of equation with partial fractional derivative of Riemann-Liouville and boundary condition that contains the generalized operator of fractional integro-differentiation. The uniqueness theorem for the solution of the problem is proved on the basis of the principle of optimality for a nonlocal parabolic equation and the principle of extremum for the operators of fractional differentiation in the sense of Riemann-Liouville. The proof of the existence of solutions is equivalent to the problem of solvability of differential equations of fractional order. The solution is obtained in explicit form.
\end{abstract}

Keywords: boundary value problem, generalized fractional integro-differentiation operator, Gauss hypergeometric function, fractional differential equation.

Received: $30^{\text {th }}$ June, $2016 /$ Revised: $8^{\text {th }}$ October, $2016 /$

Accepted: $9^{\text {th }}$ December, $2016 /$ First online:

Competing interests. We have no competing interests.

Authors' contributions and responsibilities. Each author has participated in the article concept development and in the manuscript writing. The authors are absolutely responsible for submitting the final manuscript in print. Each author has approved the final version of manuscript.

Funding. The research has not had any sponsorship.

\section{Article}

() (1) The content is published under the terms of the Creative Commons Attribution 4.0 International License (http://creativecommons.org/licenses/by/4.0/)

Please cite this article in press as:

Tarasenko A. V., Egorova I. P. On nonlocal problem with fractional Riemann-Liouville derivatives for a mixed-type equation, Vestn. Samar. Gos. Tekhn. Univ., Ser. Fiz.-Mat. Nauki [J. Samara State Tech. Univ., Ser. Phys. Math. Sci.], 2017, vol. 21, no. 1, pp. 112-121. doi: 10.14498/vsgtu1499 (In Russian).

\section{Author's Details:}

Anna V. Tarasenko (1) http://orcid.org/0000-0002-0487-8262

Cand. Phys. \& Math. Sci.; Associate Professor; Dept. of Higher Mathematics of the Architectural Engineering Institute; e-mail: tarasenko.a.v@mail.ru

Irina P. Egorova (D) http://orcid.org/0000-0001-6023-1466

Cand. Phys. \& Math. Sci.; Associate Professor; Dept. of Higher Mathematics of the Architectural Engineering Institute; e-mail: ira.egorova81@yandex.ru 


\section{References}

1. Samko St. G., Kilbas A. A., Marichev O. I. Fractional integrals and derivatives: theory and applications. New York, NY, Gordon and Breach, 1993, xxxvi+976 pp.

2. Saigo M. A remark on integral operators involving the Gauss hypergeometric function, Math. Rep. Coll. Gen. Educ., Kyushu Univ., 1978, vol.11, no. 2, pp. 135-143.

3. Kilbas A. A., Repin O. A. An Analog of the Bitsadze-Samarskii Problem for a Mixed Type Equation with a Fractional Derivative, Differ. Equ., 2003, vol. 39, no. 5, pp. 674-680. doi: $10.1023 / \mathrm{A}: 1026194020442$.

4. Gekkieva S. Kh. An analog of the Tricomi problem for a mixed type equation with a partial fractional derivative, Izvestiya Kabardino-Balkarskaya Nauchnoogo Tsentra RAN, 2001, no. 2(7), pp. 78-80 (In Russian).

5. Kilbas A. A., Repin O. A. Analog of the Tricomi problem for differential equations with partial derivatives containing fractional diffusion equation, Dokl. AMAN, 2010, vol. 12, no. 1, pp. 31-39 (In Russian).

6. Pskhu A. V. Uravneniya $v$ chastnykh proizvodnykh drobnogo poryadka [Partial Differential Equations of Fractional Order]. Moscow, Nauka, 2005, 199 pp. (In Russian)

7. Smirnov M. M. Vyrozhdaiushchiesia ellipticheskie i giperbolicheskie uravneniia [Degenerate Elliptic and Hyperbolic Equation]. Moscow, Nauka, 1966, 292 pp. (In Russian)

8. Nakhushev A. M. Drobnoe ischislenie ego primenenie [Fractional Calculation and its Application]. Moscow, Fizmatlit, 2009, 272 pp. (In Russian)

9. Nakhusheva V. A. Differentsial'nye uravneniia matematicheskikh modelei nelokal'nykh protsessov [Differential Equations of Mathematical Models of Non-Local Processes]. Moscow, Nauka, 2006, 173 pp. (In Russian) 